This item was submitted to Loughborough's Research Repository by the author.

Items in Figshare are protected by copyright, with all rights reserved, unless otherwise indicated.

\title{
Microstructural and mechanical characteristics of PHEMA-based nanofibre- reinforced hydrogel under compression
}

PLEASE CITE THE PUBLISHED VERSION

http://dx.doi.org/10.1016/j.compositesb.2015.02.033

\section{PUBLISHER}

(C) Elsevier

VERSION

AM (Accepted Manuscript)

\section{PUBLISHER STATEMENT}

This work is made available according to the conditions of the Creative Commons Attribution-NonCommercialNoDerivatives 4.0 International (CC BY-NC-ND 4.0) licence. Full details of this licence are available at: https://creativecommons.org/licenses/by-nc-nd/4.0/

\section{LICENCE}

CC BY-NC-ND 4.0

\section{REPOSITORY RECORD}

Zhao, Weiwei, Zhijun Shi, Xiuli Chen, Guang Yang, Cristina Lenardi, and Changqing Liu. 2019.

"Microstructural and Mechanical Characteristics of Phema-based Nanofibre-reinforced Hydrogel Under Compression". figshare. https://hdl.handle.net/2134/16941. 


\title{
Microstructural and Mechanical Characteristics of PHEMA- based Nanofibre-reinforced Hydrogel under Compression
}

\author{
Weiwei Zhao ${ }^{1,2}$, Zhijun $\mathrm{Shi}^{3}$, Xiuli Chen ${ }^{3}$, Guang Yang ${ }^{3}$, Cristina Lenardi ${ }^{4}$, Changqing Liu ${ }^{2, *}$. \\ ${ }^{1}$ Wuhan University of Technology, Wuhan, Hubei, 430070, China. \\ ${ }^{2}$ Loughborough University, Loughborough, Leicestershire, LE11 3TU, UK. \\ ${ }^{3}$ Huazhong University of Science and Technology, Wuhan, Hubei, 430074, China. \\ ${ }^{4}$ University of Milan, Milan, 20133, Italy. \\ *Corresponding to: Prof. Changqing Liu (C.Liu@lboro.ac.uk)
}

\section{Abstract}

Natural network-structured hydrogels (e.g. bacterial cellulose (BC)) can be synthesised with specific artificial hydrogels (e.g. poly(2-hydroxyethyl methacrylate)(PHEMA)) to form a tougher and stronger nanofibre-reinforced composite hydrogel, which possesses micro- and nano-porous structure. These synthetic hydrogels exhibit a number of advantages for biomedical applications, such as good biocompatibility and better permeability for molecules to pass through. In this paper, the mechanical properties of this nanofibre-reinforced hydrogel containing BC and PHEMA have been characterised in terms of their tangent modulus and fracture stress/strain by uniaxial compressive testing. Numerical simulations based on Mooney-Rivlin hyperelastic theory are also conducted to understand the internal stress distribution and possible failure of the nanofibre-reinforced hydrogel under compression. By comparing the mechanical characteristics of BC, PHEMA, and PHEMA-based nanofibre reinforced hydrogel (BC-PHEMA) under the compression, it is possible to develop a suitable scaffold for tissue engineering on the basis of fundamental understanding of mechanical and fracture behaviours of nanofibre-reinforced hydrogels.

Keywords: A.3-Dimensional reinforcement; B. Fracture; B. Mechanical properties; C. Finite element analysis (FEA); C. Computational Modelling. 


\section{Introduction}

Hydrogels, categorised as two groups based on their natural and artificial origins, possesses rubber-like mechanical properties ${ }^{1}$. For over five decades, hydrogels as one of the most viable biomaterials are used in biomedical disciplines, for instance, surgical sealant films or soft tissue implant. Excellent biocompatibility and suitable mechanical strength are seen as the primary advantages offered by natural hydrogels (e.g. collagen ${ }^{2}$, fibrin $^{3}$ ) in these applications. However, there exist some issues, such as high cost, limited natural source, and risk of contamination. Artificial synthesised hydrogels are finding more uses due to the low cost and highly reproducible with controlled chemical structure, but tend to possess poor biocompatibility and low toughness. For example, the maximum tangent modulus of the most tough synthesised hydrogel (e.g. PHEMA) is $2 \sim 4$ $\mathrm{MPa}$, which is still much lower than some natural tissues, e.g. articular cartilage ${ }^{4}$. In order to improve biocompatibility, mechanical strength and allow low cost fabrication, a number of routes to improve the toughness of gels by modifying hydrogels have been reported in literature ${ }^{5,6,7,8}$, including the uses of micro- and nano-fibres to reinforce the hydrogels ${ }^{9,10}$.

Nanofibre-reinforced hydrogel as a tough and strong gel matrix is physically synthesised by blending two types of hydrogels, e.g. PHEMA and bacterial cellulose. In such case, original hydrogel usually acts as micro- or nanofibres to achieve the reinforcement of the composite hydrogel, by constructing its internal network structure similar to the reinforced concrete. The nanofibres can be short fibres like woven ${ }^{11}$, polymer fibrils formed by polyvinyl alcohol ${ }^{12}$, or bacterial cellulose ${ }^{13}$. Such fibre-reinforced hydrogels can exhibit high strength, high tolerance to tensile strain, and good fracture toughness. There have been some experimental investigations on such synthesised hydrogels, however, theoretical analysis are highly demanded to elaborate the detailed mechanism of fracture of the nanofibre-reinforced hydrogel, for instance, through numerical method.

In the present study, bacterial cellulose (BC), an ultrafine-fibre network produced by bacteria of genus Acetobacter ${ }^{14,15}$, has been utilised as nano-fibrous scaffold to be blended with PHEMA, aiming to establish a novel kind of nanofibre-reinforced hydrogel with enhanced mechanical strength. The mechanical characteristics of the nanofibre-reinforced hydrogel, in terms of the stress-strain correlation, the relationship between tangent modulus and strain, and the fracture mechanism, are 
thereby investigated through experiments and numerical simulation. Using finite element analysis (FEA) technique ${ }^{16}$, the developed model based on Mooney-Rivlin theory (hyperelasticity) was also validated by experimental results to simulate the network structure of composite hydrogel, in order to understand the fracture mechanism of the reinforced hydrogel.

\section{Methodology}

\subsection{Sample preparation}

Preparation of Bacterial Cellulose: Acetobacter Xylinum ATCC53582 was statically cultured within Hestrin and Schramm medium under $30^{\circ} \mathrm{C}$ for 20 days. The Hestrin and Schramm medium consists of D-glucose $(20 \mathrm{~g} / \mathrm{L})$, peptone $(5 \mathrm{~g} / \mathrm{L})$, yeast extract $(5 \mathrm{~g} / \mathrm{L})$, dibasic sodium phosphate $(2.7 \mathrm{~g} / \mathrm{L})$ and citric acid (1.5 g/L). After the culturing process, the obtained bacterial cellulose hydrogel was soaked in distilled water for 48 hours after the extraction from the culture medium. Finally, the bacterial cellulose hydrogel was boiled in the solution of sodium hydroxide (1\% weight of water) for $30 \mathrm{~min}$, then washed by distilled water again for 2 hours in order to make the hydrogel reach a neutral $\mathrm{pH}$ value.

Synthesis of nanofibre-reinforced hydrogel: The PHEMA hydrogel material was prepared based on the same chemical reagents as that was reported in the literatures ${ }^{17,18}$. It is synthesised using HEMA solution (70 wt $\%$ of hydroxyethyl methacrylate and $30 \mathrm{wt} \%$ of water solvent), cross-linker EGDMA (0.1 mol\% of HEMA) and catalyzer TEMED (1.4\% weight of HEMA). Before the polymerization, the specimen of BC was immersed in this mixed HEMA solution for at least 48 hours, which can allow the HEMA solution to fill the nano-gaps between fibres of $\mathrm{BC}$. Then, the sample of $\mathrm{BC}$ which was fully filled with HEMA was immersed into the ammonium persulfate solution (20\% weight of water), to initiate the polymerisation of HEMA. The polymerising reaction was completed by $60 \%$ within one hour ${ }^{19,20}$, and was finished until the catalyzer TEMED was fully consumed during the formation of chains of PHEMA around the nanofibre of BC.

Swelling and washing of nanofibre-reinforced hydrogel: After the synthesis of the BC-PHEMA nanofibre-reinforced hydrogel, a swelling and washing process had to be performed releasing the constraint between PHEMA chains after reaction and the removal of the water-soluble contaminations 
or incomplete reacted molecules, respectively ${ }^{21}$. The nanofibre-reinforced hydrogel specimen was firstly sunk into one litre of 50 vol\% ethanol aqueous solution for 4 hours, then removed from 50 vol\% ethanol solution and immediately transferred into 40 vol\% ethanol solution for another 4 hours to dilute the ethanol sucked in the specimen. This process was repeated by decreasing the concentration of ethanol solution by 10 vol\% each time until ethanol content in the solution reached zero. This process can fulfil the dual purpose of both swelling and washing of the hydrogel specimen at the same time.

Freeze-drying of hydrogel specimens: The above prepared hydrogels have to be fully dried prior to the SEM observation. Air-drying of the polymers, especially hydrogels, causes collapse and shrinkage of specimen at the gas-liquid interface. Thus, laboratory freeze-drying equipment (FD-1-50, Boyikang Ltd.) was used in this study to remove the water content in the specimens. The water content are firstly frozen together with the specimen, then removed in a supercritical state by high degree of vacuum, so that no gas-liquid interface present within the specimen during the drying. Thus, drying without collapse and shrinkage of specimen can be achieved.

\subsection{Mechanical compression}

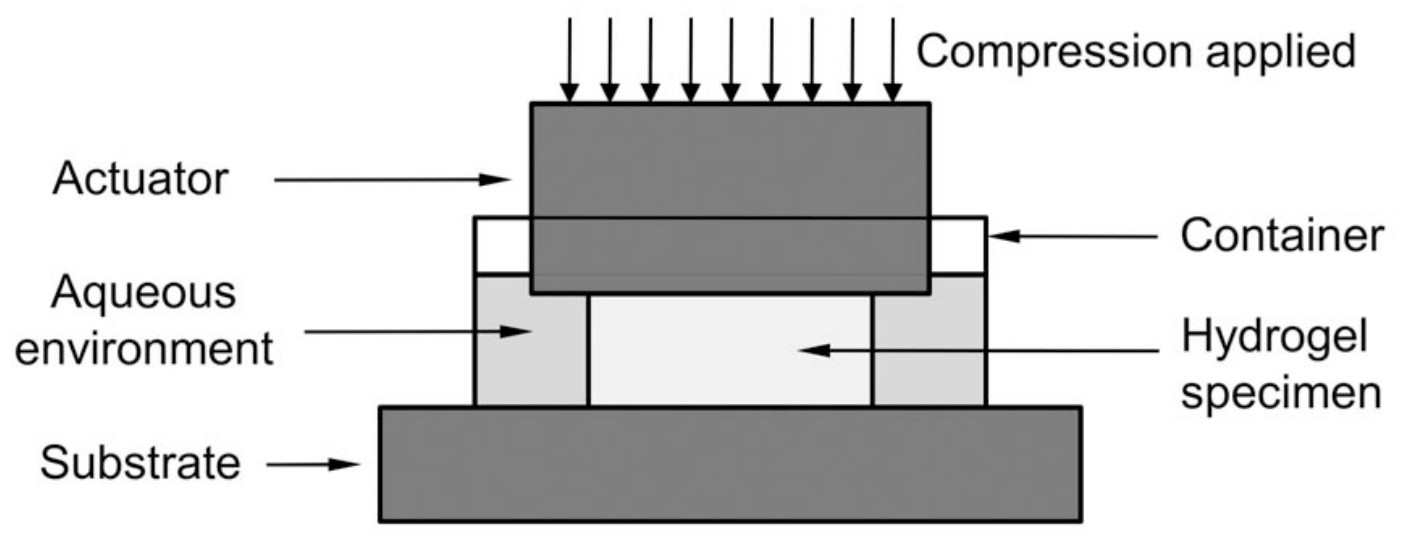

Figure 1. Schematic illustration of compressive testing of hydrogel specimens immersed in aqueous solution to keep a constant swelling environment.

An Instron Series 3366 Model of compressive test apparatus with $2 \mathrm{kN}$ load cell was used to carry out mechanical compression tests. The error of measured displacement was approximately $\pm 0.05 \%$, and the error of detected load was within $\pm 0.5 \%$. The testing configuration was designed to obtain the stress-strain curve and ultimate strength of BC, PHEMA and the nanofibre-reinforced hydrogel (BC- 
PHEMA) specimens under compression. The hydrogels were prepared and cut into cylindrical specimens of $20 \mathrm{~mm}$ diameter and $10 \mathrm{~mm}$ height. As is shown in Figure 1, the specimen was placed in a container filled with diluted water, as an aqueous environment during the testing which guarantees the constant swelling and hydration conditions of hydrogel, e.g. fully swollen state. The compressive speed $0.5 \mathrm{~mm} / \mathrm{s}$ was used with a $0.05 \mathrm{~N}$ preload being applied on the test specimen.

\subsection{Numerical simulation}

Numerical simulation was carried out to establish predictive model on mechanical behaviour of hydrogels using COMSOL Multiphysics software (Burlington, MA, USA). The core of the simulation is the theoretical model for the materials, which is used to understand the mechanical behaviour of hydrogels.

In theory, hydrogels can be regarded as an incompressible hyperelastic material ${ }^{22}$, like an ideal rubber which is highly deformable and recoverable without volume change during deformation. According to the simulation theories for hydrogel in literature ${ }^{23,24}$, Mooney-Rivlin Theory ${ }^{25,26}$ was best used theoretical basis for the description of the hyperelastic compressive behaviour of hydrogels. In this work, Mooney-Rivlin model was also employed to predict the mechanical deformation of BC, PHEMA and the nanofibre-reinforced hydrogel (BC-PHEMA). The constitutive formula ${ }^{26}$ of MooneyRivlin model is therefore given by:

$$
W\left(I_{1}, I_{2}\right)=C_{10}\left(I_{1}-3\right)+C_{01}\left(I_{2}-3\right)
$$

where, $W$ denotes the strain energy density function; $I_{1}$ and $I_{2}$ are first and second invariant of the unimodular component of the left Cauchy-Green deformation tensor. While $C_{10}$ and $C_{01}$, are the temperature-dependent constants which can be derived by analysing the stress-strain data from uniaxial compression or tension test. By replacing the Cauchy-Green deformation tensor with engineering stress $(\sigma)$ and stretch ratio $(\lambda)$, the constitutive formula of Mooney-Rivlin model can be derived as:

$$
\frac{\sigma}{2\left(\lambda-\lambda^{-2}\right)}=C_{10}+\frac{C_{01}}{\lambda}
$$

Hence, Mooney-Rivlin model of a hyperelastic material can be determined based on Equation (2) where the engineering stress and stretch ratio can be obtained through compressive or tensile testing. 


\section{Results and Discussion}

\subsection{Microstructural Characteristics of PHEMA-based Natural Nanofibre-reinforced}

Hydrogel

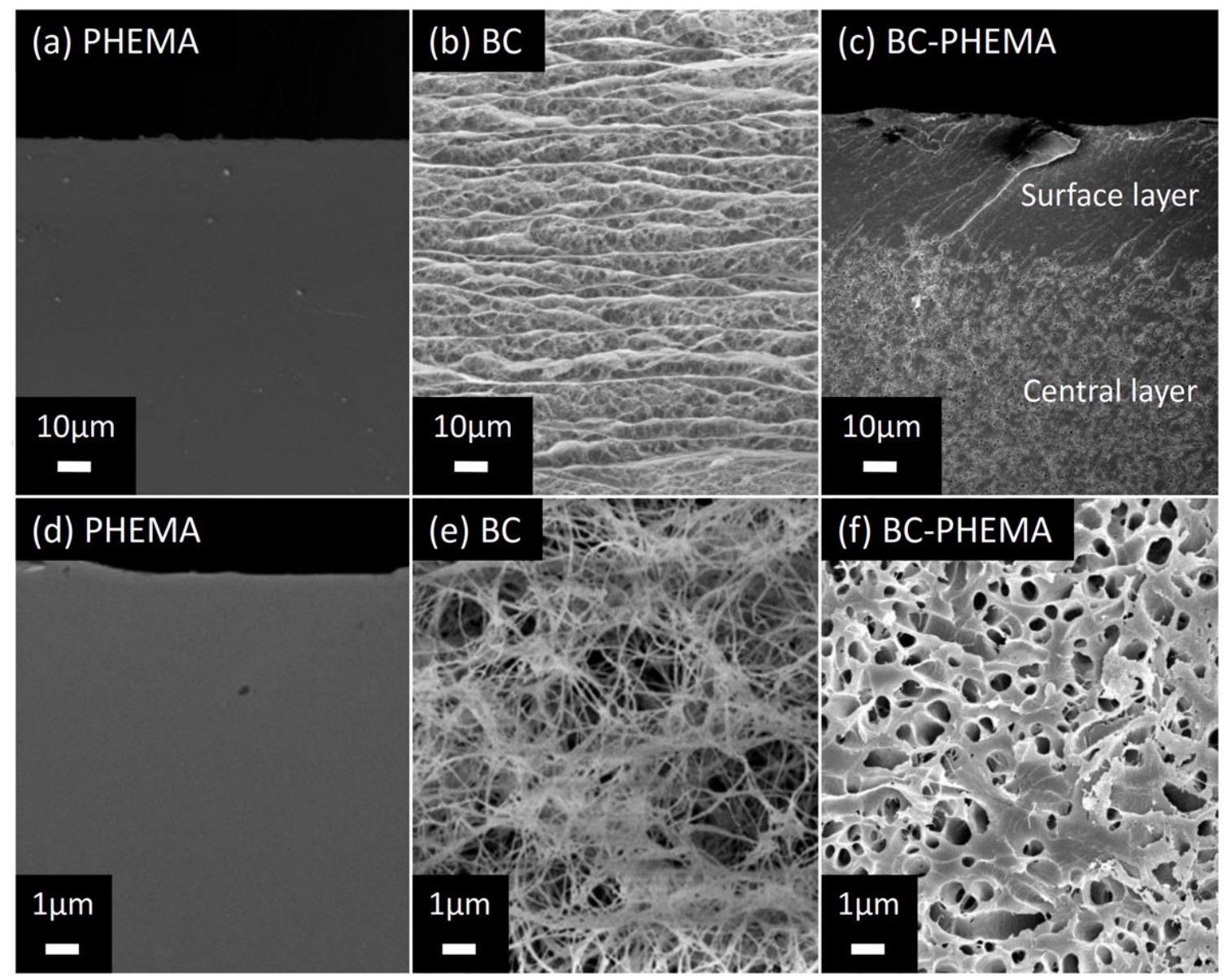

Figure 2. Cross-sectional view of PHEMA $(a, d), B C(b, e)$ and BC-PHEMA $(c, f)$ hydrogel specimens by Scanning electron microscope. (f) is the enlarged central layer which is shown in (c). PHEMA has high compactness at microscale; $\mathrm{BC}$ is a network constructed by nanofibres; BC-PHEMA is a porous structure.

The microstructures of the fabricated BC-PHEMA hydrogel and its components BC and PHEMA hydrogel were examined by SEM, and the results are shown in Figure 2. From Figure $2 \mathrm{a} \& 2 \mathrm{~d}$, the microstructure of PHEMA hydrogel exhibits good uniformity and high compactness, because PHEMA hydrogel is polymerized from homogeneous aqueous solution with discrete uniform density. However, according to Figure $2 \mathrm{~b} \& 2 \mathrm{e}, \mathrm{BC}$ can be characterized as an ultrafine network structure. The distance between fibres within the cellulose network are in a range from $100 \mathrm{~nm}$ to $1 \mu \mathrm{m}$, which is in a good agreement with the data reported in literature ${ }^{27,28}$. Interestingly, the composite nanofibre-reinforced hydrogel consists of two layers, surface layer and central layer shown in Figure 2c. The two layers 
exhibit quite distinct textures. According to an investigation on the texture of $\mathrm{BC}^{27}$, the surface layer was formed at the interface between culture broth and the external environment (air) during culture process, and characterised as compact cellulose network structure which is more concentrated than the networks of the central layer. Iguchi et al. ${ }^{29}$ explained the function of the surface layer that bacteria intend to construct a 'cage' to protect themselves from heavy-metal ions or other kind of bacteria, but still leave small gaps allowing nutrients (small molecules) can-to be supplied easily by diffusion. Whereas, the central layer is constructed less concentrated because it acts as scaffolds allowing bacteria to attach, proliferate and spread. In this study, the thickness of this surface layer increases through the polymerisation of PHEMA around the nanofibres of BC. Beneath the surface layer approximately $30 \mu \mathrm{m}$ thick, the composited hydrogel has microporous structure inside the central layer. The formation of these micropores is attributed to the gas bubbles involved during its culturing process, prior to the synthesis of the reinforced hydrogel. The size and density of bubbles are-both depending on the size and distribution of the microporous structure of BC. Treatment of the sample with in the vacuum before the polymerisation progress; will helpcan reduceing the numbers of bubbles, thereby achieving the better control of the final porosity of composited reinforced hydrogel BCPHEMA. When the $B C$ is immersed into the HEMA solution, HEMA solution will infiltrate the $B C$ network from the surface to inside of the structure. Such infiltration is driven by the capillary pressure and osmotic pressure because HEMA solution and BC are both hydrophilic. At the same time, gas bubbles deep-inside the BC network will prevent the further infiltration of HEMA solution. Thus, HEMA solution cannot completely fill the micropores deep-inside BC, but still infiltrate into the inside BC along the nanofibres, until HEMA solution reaches saturation inside BC. After the reaction of polymerization, the porous structure of the reinforced hydrogel is formed by the solidified PHEMA around the nano-fibrous scaffold of $\mathrm{BC}$.

In order to quantify the size of the pores in the central layer of composited reinforced hydrogel (BC-PHEMA), a statistical analysis has been carried out, based on the diameter values of 288 pores which are measured from SEM photographs. Figure 3a illustrates the distribution of the diameter values. Accordingly, over $76 \%$ pores in the reinforced hydrogel fall primarily in the range of $0.45 \sim 0.65$ $\mu \mathrm{m}$, with an average value of $0.57 \mu \mathrm{m}$, which represents the quantified size of the micropores in the central layer of the composited reinforced hydrogel. Thus, the porosity of the central layer of the composited reinforced hydrogel can be figured outestimated as $4.51 \%$, which has a good match with 
the porosity values of mandibular condyle $(3.5 \%)^{30}$, and femoral shaft (approximately $\left.5 \%\right)^{31}$.
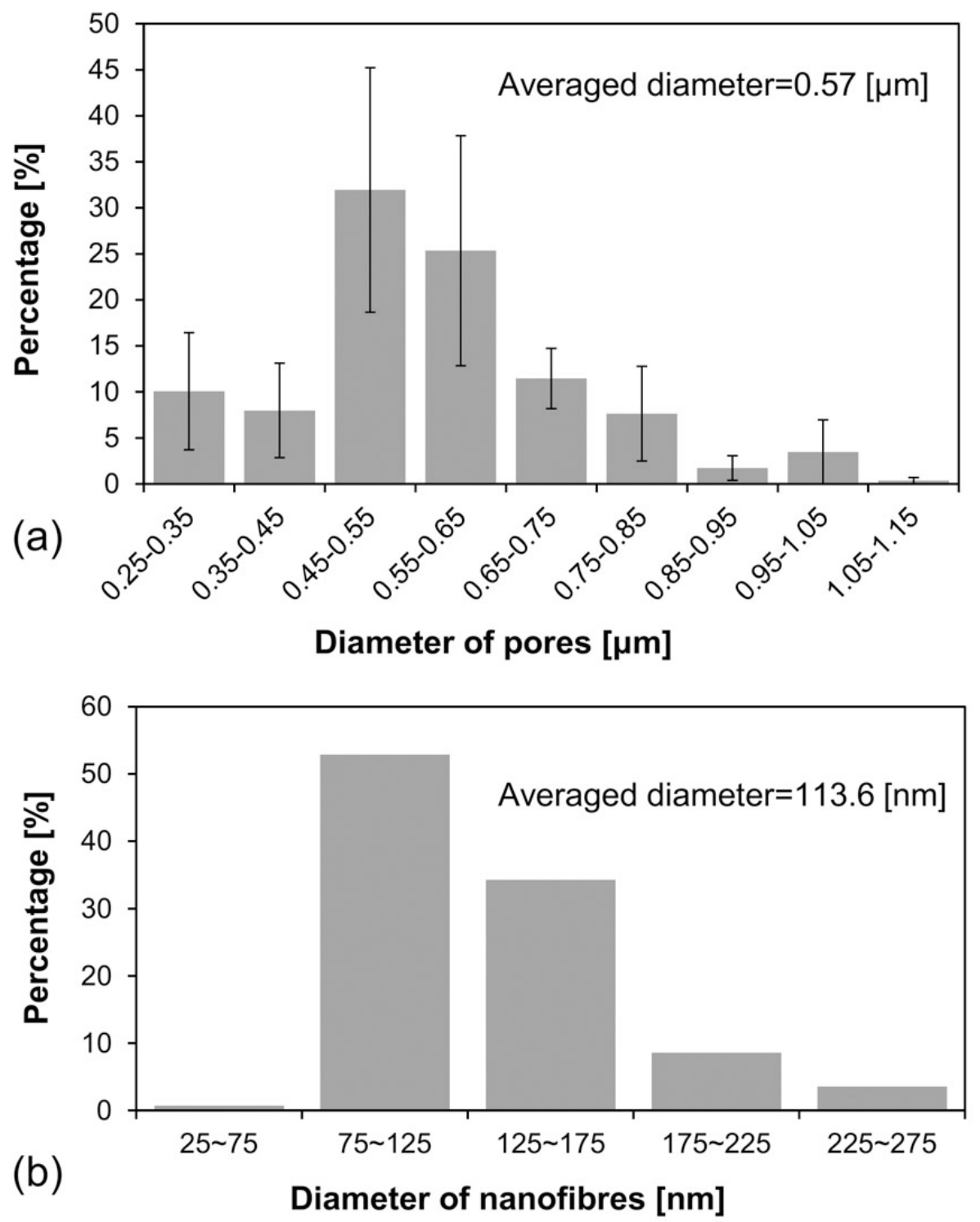

Figure 3. (a) The distribution of the micropore sizes. Diameter data were collected based on 288 pores from the SEM photograph of the reinforced hydrogel. (b) The distribution of the nanofibre diameters. Diameter data were collected based on 140 nanofibres from the SEM photographs of the Bacterial Cellulose (BC). Such results have strong agreement with the work ${ }^{32}$ by Ying Li, et al.

\subsection{Mechanical Characteristics of PHEMA-based Natural Nanofibre-reinforced} Hydrogel

To investigate the mechanical characteristics of the reinforced hydrogel and to examine the reproducibility of the results from compressive tests, three groups of experimental compressive test have been carried out on the specimens with the same dimensions. The stress-strain curves for the reinforced hydrogel BC-PHEMA specimens are presented in Figure 4a, with the corresponding tangent modulus (also known as instantaneous modulus ${ }^{33}$ ), being estimated to the first derivative of 
the stress-strain curve at each data point, as plotted in Figure $4 \mathrm{~b}$. The value of each point of tangent modulus in Figure $4 \mathrm{~b}$ is derived by smoothing every 3 data points nearby, based on the method reported by Screen $\mathrm{H}$. et al. ${ }^{34}$. At a compressive strain $(\varepsilon)$ varying from zero to $40 \%$, from Figure $4 a$, the hydrogel samples of each test exhibit identical nonlinear behaviour. As the compressive strain is further increased, the hydrogel specimens begin to reach their ultimate strength and a point of fracture. The strains that have caused failures are predominantly in the range of $0.46 \sim 0.48$, which corresponds to a range of failure stresses within 1.76 1.90 MPa.

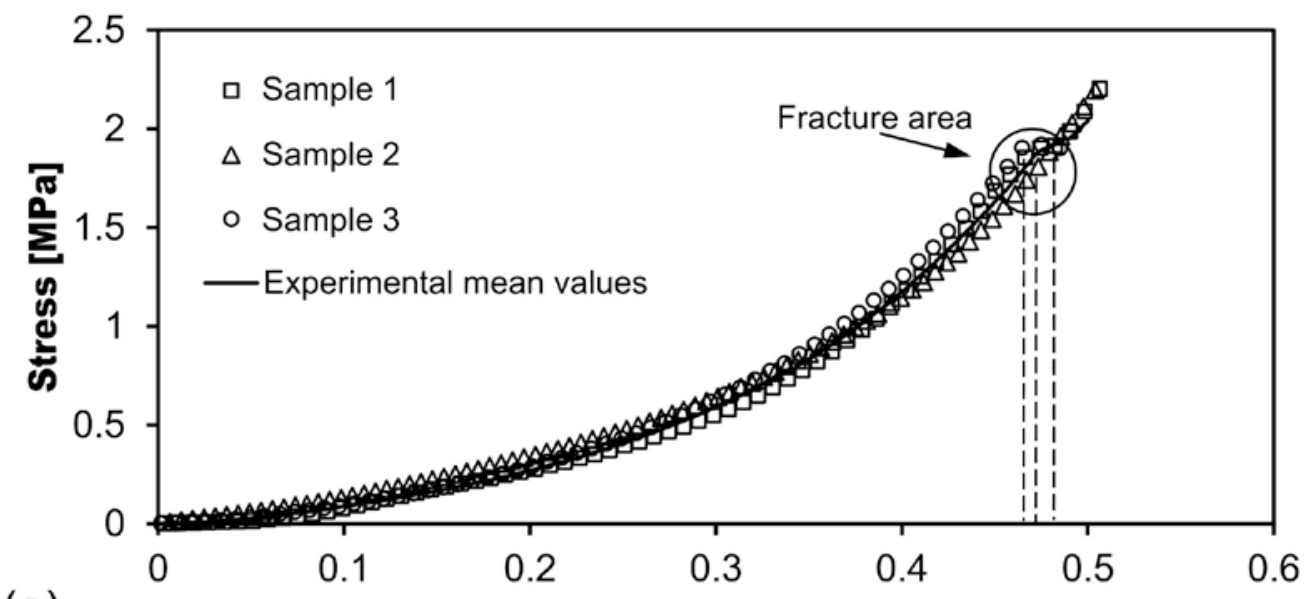

(a)

Strain

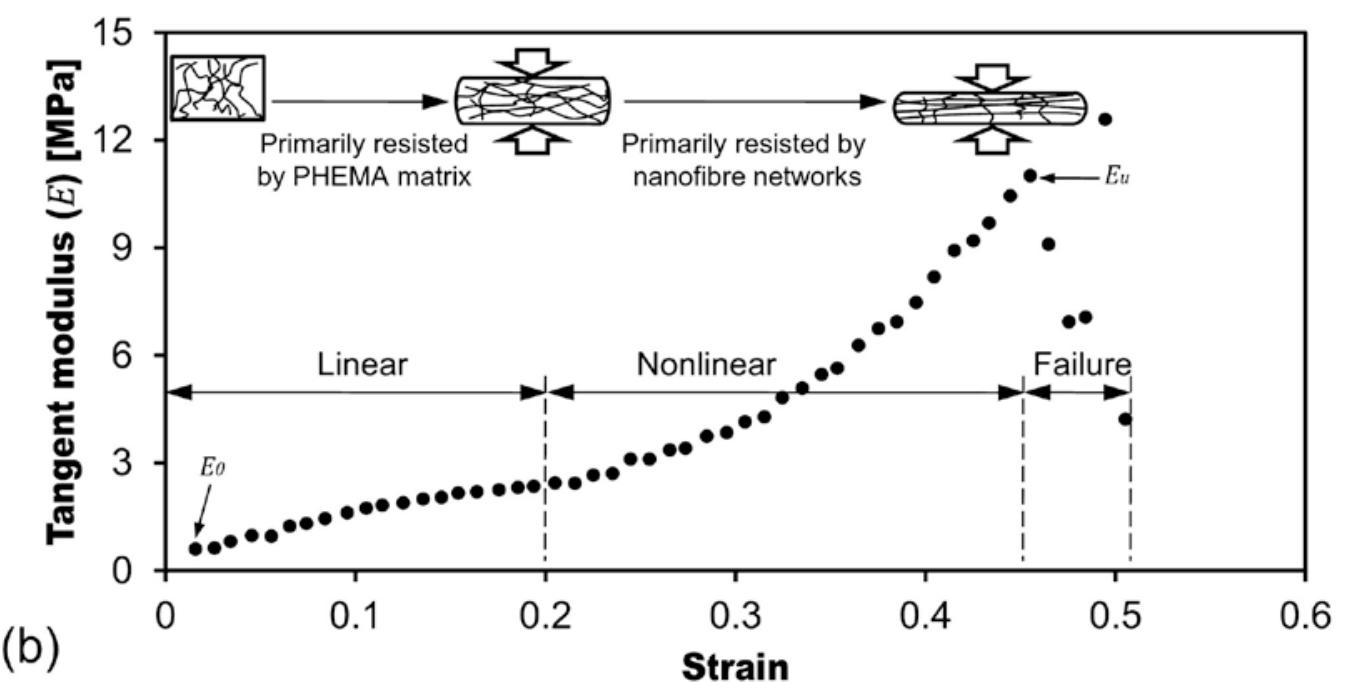

Figure 4. (a) Compressive stress versus deformation strain of five different identically sized specimen of the reinforced hydrogel (BC-PHEMA). The breaking strain locates around 0.47. (b) Tangent modulus with respect to strain. During the linear increase stage, the compression is primarily resisted by PHEMA. In the nonlinear increase stage, the compression is primarily resisted by $\mathrm{BC}$, the nanofibre network.

According to Figure $4 \mathrm{~b}$, before the material fractured, the tangent modulus $(E)$ increases with the increase of strain. It indicated the nonlinear deformation characteristics of BC-PHEMA specimen 
under compression. The tangent modulus $E$ increases from $0.97 \mathrm{MPa}\left(E_{0}\right)$ (at $\left.\varepsilon=0.01\right)$ to $11.56 \mathrm{MPa}$ $\left(E_{u}\right)$ (at $\left.\varepsilon=0.45\right)$, which is the maximum strain that the material can subdue before fracture. These tangent modulus are within the strength range of articular cartilage $(4 \sim 10 \mathrm{MPa})^{35,36}$ indicating that reinforce hydrogel can meet the requirement of mechanical strength as a replacement of articular cartilage. Besides, three distinct regions can be identified from the curve of tangent modulus to strain: 1) linear increase at initial stage, 2) nonlinear increase at the second stage, and 3) irregular decrease after failure stage. Before compression $(\varepsilon=0)$, the nanofibres of BC material were at their relaxing state and embraced by PHEMA. In the compressive strain range of $0 \sim 0.2$, the nanofibre network was compressed in axial direction and stretched in radial direction in the cylindrical specimen. The compression can only cause the deformation of the nanofibre network from their relaxing state to stressing state, causing resist force against the compression. The compression is primarily endured by PHEMA at this stage. As the compressive strain is further increased, nanofibres in the bulk material were completely under the stressed state. In the strain range of $0.2 \sim 0.5$, the resist force against the compression was mainly induced by the nanofibre networks, and the PHEMA matrix in the material still played an important role in resistance to the compressive force to maintain the network structure of nanofibres. If the strain is beyond the ultimate compressive strength of the BC-PHEMA material, the potential failure on both PHEMA matrix and the nanofibres originated from BC is likely to occur, thus lead to the fracture of BC-PHEMA material.

On the basis of three groups of experimental data from the compressive tests on PHEMA, BC and the reinforced hydrogel BC-PHEMA specimens, the fracture stress, fracture strain, ultimate tangent modulus and initial tangent modulus are summarised in Table 1 respectively. PHEMA, as one of the strongest artificial synthesised hydrogels which is highly resistant to compression, gives a large initial tangent modulus $E_{0}$ (Young's modulus) about $0.85 \mathrm{MPa}$. During the compressive test, the tangent modulus increases with the increase of strain due to the hyperelastic responses of PHEMA material, and reaches the peak value $E_{u}$ at approximately $2.48 \mathrm{MPa}$ near the strain at 0.5 , prior to the fracture of the fracture of the specimens. As for pure BC specimens, no fracture stress and strain was observed during the compressive test, because the limit of the applied maximum load $(2 \mathrm{kN})$ which is smaller than the fracture strength of $\mathrm{BC}$. However, it is apparent to see that both $E_{0}$ and $E_{u}$ values from the $\mathrm{BC}$ specimens are much smaller than those of PHEMA. These results indicate that BC is highly deformable but much softer than PHEMA. According to the results for BC-PHEMA from Table 1, the 
initial tangent modulus $E_{0}$ of BC-PHEMA is higher than those of both PHEMA and BC, especially almost 8 times larger than BC's initial tangent modulus. The BC-PHEMA hydrogel fractured at the compressive stress of $1.83 \mathrm{MPa}$, when the specimen is compressed by approximately $47 \%$ in strain. This fracture of BC-PHEMA gives an ultimate tangent modulus $E_{u}$ at around $10 \mathrm{MPa}$, which is over 3 times larger than that of PHEMA, and 20 times larger than that of BC. Thus, the results in Table 1 indicate that the BC-PHEMA hydrogel is harder and more resistant to compressive strain than both of its origins, PHEMA and $\mathrm{BC}$, because the nanofibres of $\mathrm{BC}$ reinforced the micro-structure and improved the mechanical strength of the material.

Table 1. Results from compressive tests on three groups of hydrogel specimens

\begin{tabular}{lcccc}
\hline Sample & $\begin{array}{l}\text { Fracture stress } \\
{[\mathrm{MPa}]}\end{array}$ & Fracture strain & $\begin{array}{l}\text { Ultimate tangent } \\
\text { modulus }\left(E_{u}\right)[\mathrm{MPa}]\end{array}$ & $\begin{array}{l}\text { Initial tangent } \\
\text { modulus }\left(E_{0}\right)[\mathrm{MPa}]\end{array}$ \\
\hline PHEMA & $0.53 \pm 0.03$ & $0.50 \pm 0.03$ & $2.48 \pm 0.23$ & $0.85 \pm 0.01$ \\
BC & - & - & $0.43 \pm 0.08$ & $0.11 \pm 0.01$ \\
BC-PHEMA & $1.83 \pm 0.07$ & $0.47 \pm 0.01$ & $10.01 \pm 0.42$ & $0.97 \pm 0.02$ \\
\hline
\end{tabular}

To numerically characterise the behaviour of $\mathrm{BC}$, PHEMA and BC-PHEMA hydrogel, the simulations with an aim to describing the responses of each material to compression, in terms of stress-strain curve, are established. According to literatures ${ }^{23,25}$, where the hyperelastic theory is adopted in the implementation of modelling. The Mooney-Rivlin coefficients $C_{01}$ and $C_{10}$ are individually calculated through the stress-strain curve of specimens from each hydrogel. Figure 5 affirmed that the best fittings of the stress-strain curves derived from compression tests of three types of hydrogels can be achieved by simulations in terms of their mechanical behaviour under compression. As it clearly presented, the BC-PHEMA hydrogel is the strongest among the three hydrogels, whilst the $\mathrm{BC}$ is the weakest. For instance, the stress value of the BC-PHEMA hydrogel was approximately $1.25 \mathrm{MPa}$, which is three times larger than the stress $(0.43 \mathrm{MPa})$ of PHEMA hydrogel and thirty times larger than the stress $(0.041 \mathrm{MPa})$ of $\mathrm{BC}$ at the strain of 0.4 . This significant change of mechanical properties is primarily attributed to the nanofibres of cellulose which act as interconnected networks underneath PHEMA matrix in the BC-PHEMA hydrogel thus the reinforcement of the matrix can be achieved with the high mechanical strength observed from BC-PHEMA hydrogel. However, in Figure 5, it indicates the constraints of the numerical modelling that it failed to predict the failure behaviour of the materials, and their internal characteristics due to the fracture of nanofibres or the crack of PHEMA matrix. 


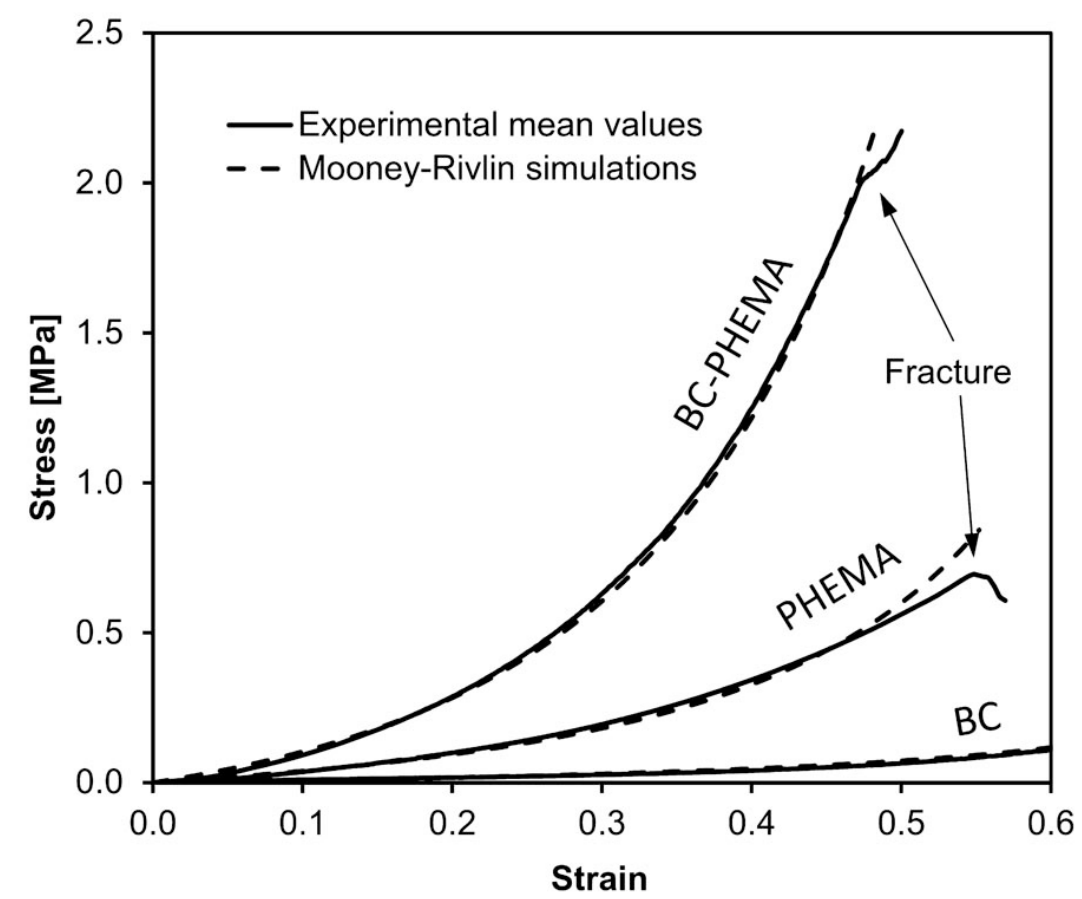

Figure 5. Comparison of uniaxial compressive test data and curve fitting through Mooney-Rivlin model based simulations, for the specimens of PHEMA, BC and reinforced hydrogel (BC-PHEMA).

To gain fundamental understanding on the failure of the BC-PHEMA hydrogel, the micro-structure which can reflect the interactions between nanofibres and PHEMA matrix under compressive conditions has been built into the numerical simulation. The geometry of modelling is based on a cubic element extracted from the schematic structure of the reinforced hydrogel which exhibits as PHEMA matrix embedded with inter-crossed nanofibres. As is illustrated in Figure $6 \mathrm{a}$, a size at $44^{\star} 4$ $\mu \mathrm{m}^{3}$ of cubic structure embracing several inter-crossed nanofibres (diameter ${ }^{32}$ at $100 \mathrm{~nm}$, also referenced to the averaged diameter of nanofibres shown in Figure $3 b$ ) is constructed in modelling. Bacterial cellulose, as the inserted nanofibres in the structure, provides a soft but compact reinforcement to the cubic PHEMA hydrogel substrate. Thus, the simulation results in terms of Von Mises stress distribution in Pascal of the nanofibres under different compressive strain at $20 \%$ and $50 \%$ are obtained and presented in Figure $6 \mathrm{c}$ and Figure $6 \mathrm{e}$, respectively. The $20 \%$ strain were selected to represent the initial turning point to the nonlinear tangent modulus and $50 \%$ is close to the fracture strain as seen in Figure 4b. To clearly illustrate the stress distribution of nanofibres, the PHEMA matrix has been made invisible. As results, the numerical simulations quantitatively evaluated that the maximum stress on the nanofibres under $20 \%$ compressive strain is $0.566 \mathrm{MPa}$ (Figure 6c), and the maximum stress on the nanofibres under $50 \%$ compressive strain is $1.585 \mathrm{MPa}$ (Figure 6e). 
(a)
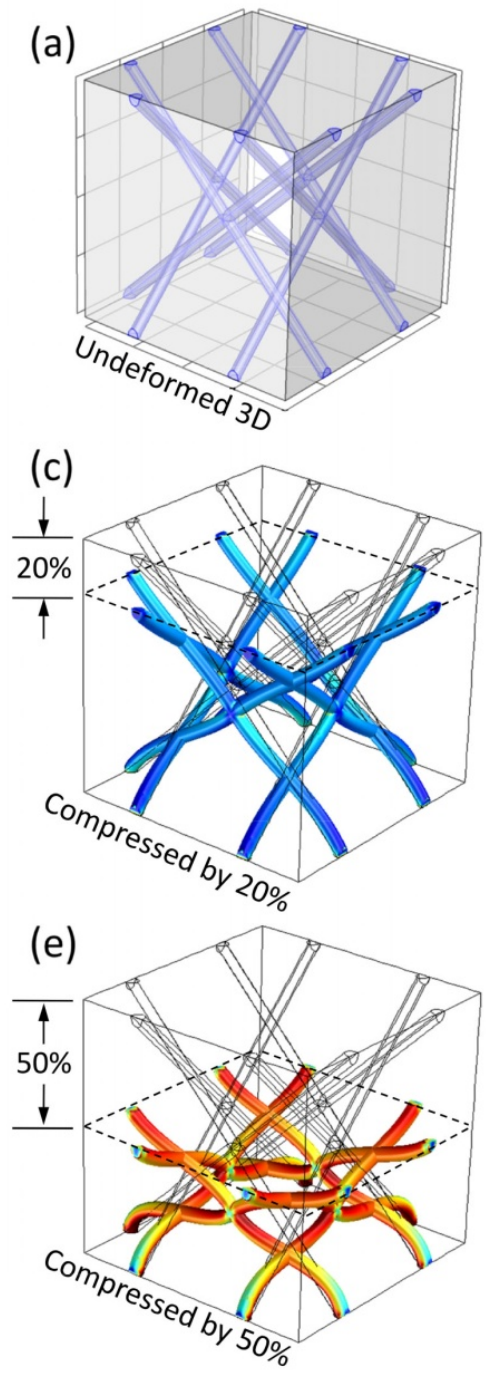

(b)

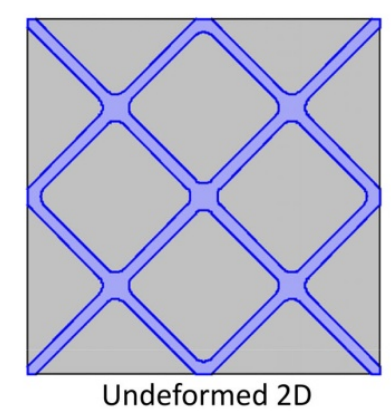

(d)

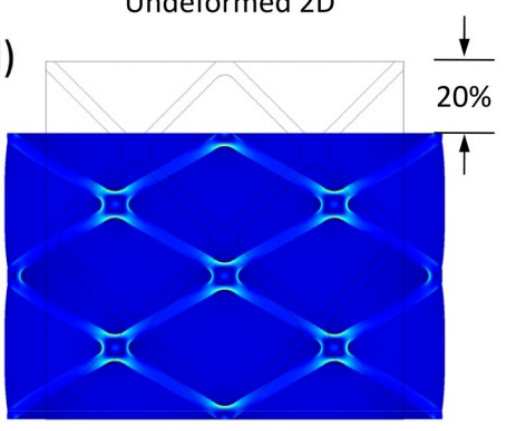

Compressed by $20 \%$

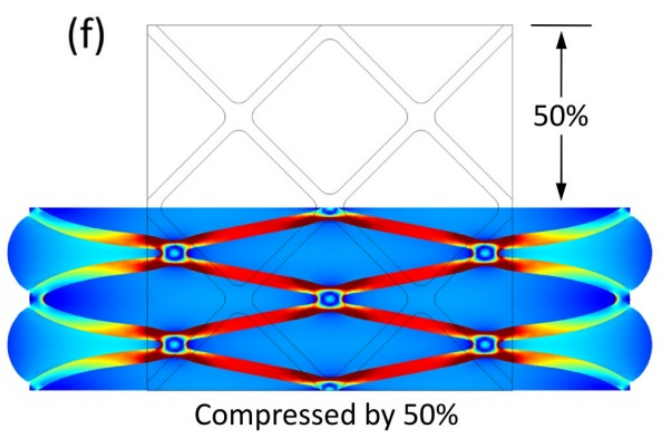

Compressed by $50 \%$

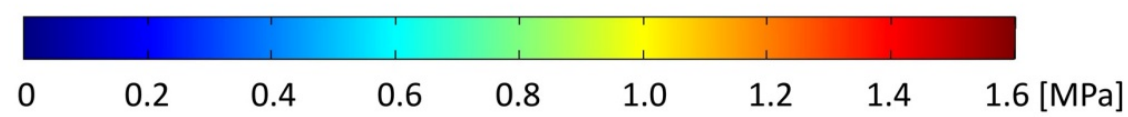

Figure 6. 3D modelling based on a cubic element from reinforced hydrogel with size of $4 * 4 * 4\left[\mu \mathrm{m}^{3}\right](a, c, e)$. $2 \mathrm{D}$ modelling based on a squared sliced element from reinforced hydrogel with size of $4^{*} 4^{*} 0.1\left[\mu^{3}\right](b, d, f)$. The original 3D and 2D structures are shown in (a) and (b) without stress or deformation. Internal stress (Von Mises) distribution in Pascal with respect to different compressive strain $20 \%$ (c\&d) and 50\% (e\&f).

Table 2. Geometry Dimensions and Simulation Results under $\mathbf{2 0 \%}$ and $\mathbf{5 0 \%}$ Strain

\begin{tabular}{lccc}
\hline & & 2D modelling & 3D modelling \\
\hline \multirow{2}{*}{ Geometry } & Dimension of matrix $\left[{\left.\mu \mathrm{m}^{3}\right]}^{2}\right.$ & $4^{*} 4$ & $4^{*} 4^{*} 4$ \\
& Dimension of fibres $[\mathrm{nm}]$ & Wide: 100 & $\varnothing 100$ \\
\hline \multirow{2}{*}{$20 \%$ strain } & Maximum stress on nanofibres [MPa] & 0.425 & 0.566 \\
& Maximum stress on PHEMA matrix [MPa] & 0.528 & - \\
\hline \multirow{2}{*}{$50 \%$ strain } & Maximum stress on nanofibres [MPa] & 1.527 & 1.585 \\
& Maximum stress on PHEMA matrix [MPa] & 0.796 & - \\
\hline
\end{tabular}


It has been found difficult to understand the interactions between PHEMA matrix and BC nanofibres based on the 3D modelling (Figure $6 a, 6 c, 6 e)$. Therefore, to gain the Von Mises stress distribution at the interface between PHEMA matrix and nanofibres, two dimensional model at size of $4 * 4 \mathrm{~m}^{2}$, with insertion of inter-crossed nanofibres are built in the numerical simulation (see Figure $6 \mathrm{~b}$ ). The simulations were performed based on the same parameters of material properties and MooneyRivlin coefficients used in 3D modelling. The geometry dimensions and simulation results are thus summarised in Table 2. In terms of the Von Mises stress distribution with respect to $20 \%$ and $50 \%$ compressive strains, the simulation results are illustrated and compared in Figure $6 \mathrm{~d}$ and $6 f$, respectively. According to the colour range shown in Figure $6 \mathrm{~d}$, it is noticeable that the stress is evenly distributed in both PHEMA matrix and nanofibres under $20 \%$ compressive strain, though it is slightly concentrated at the inter-cross junctions of nanofibres. However, under $50 \%$ compressive strain in Figure $6 \mathrm{f}$, the stress is mainly applied on the nanofibres and related junctions, thereby resulted in large stress differences between the nanofibres and PHEMA matrix. This large stress gradient shown in Figure $6 \mathrm{f}$ is also indicated from the Table 2 that the maximum stress on nanofibres (1.527 MPa) is nearly two times larger than that on PHEMA matrix (0.796 MPa) under $50 \%$ compressive strain. Due to this large stress differences, the fracture of the reinforced hydrogel BC-PHEMA was probably initiated at the interface between nanofibres and PHEMA matrix. This result implies that within the strain range $0 \sim 20 \%$, the resistant force against the compression is primarily facilitated by PHEMA, but assisted by nanofibres. However, within the strain range $20 \% \sim 50 \%$, PHEMA matrix is likely to reach its ultimate strength, as such the compression was primarily withheld by nanofibres.

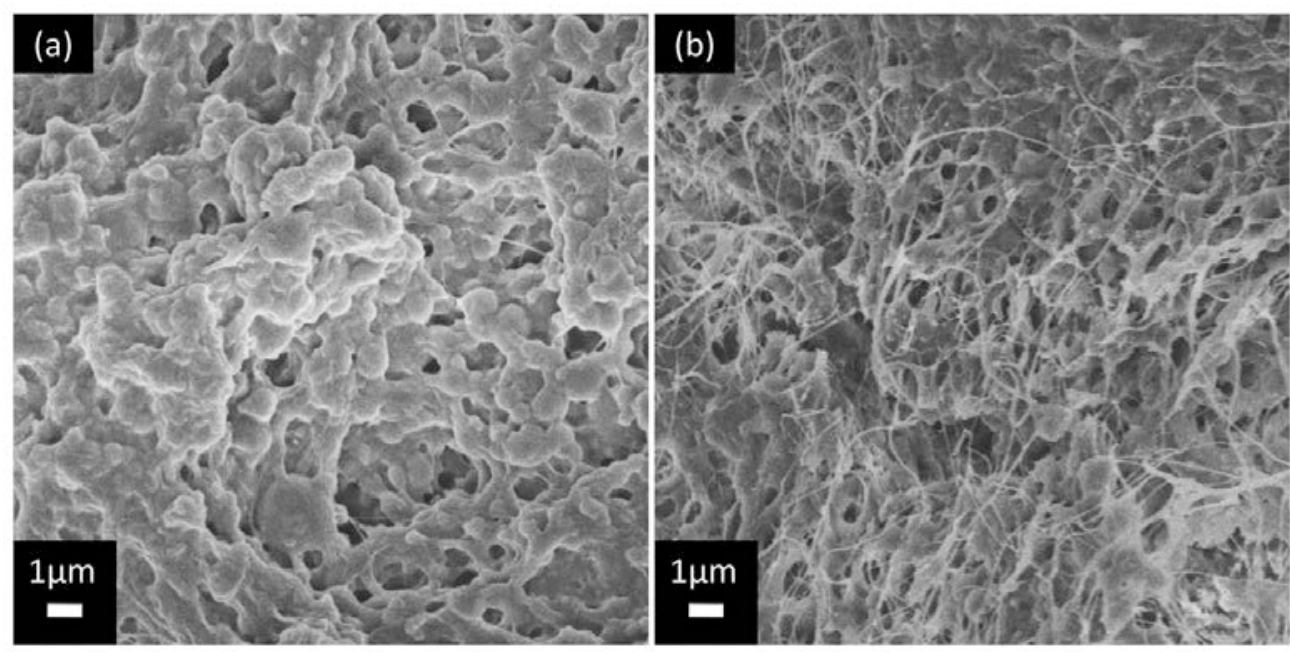

Figure 7. Scanning electron micrographs of compressed reinforce hydrogel in (a) $20 \%$ compressive strain and (b) $50 \%$ compressive strain with material failure. Sample preparation via freeze drying process. 
According to the Fracture stress of PHEMA hydrogel at approximately 0.53 strain, which is summarised in Table 1, it can be found that the failure of the PHEMA matrix in BC-PHEMA material is potentially occurred below $20 \%$ compressive strain based on the results from Table 2 . When the strains are over $20 \%$, nanofibre networks play a significant role in the strengthening of BC-PHEMA hydrogel. Thus, the failure of the BC-PHEMA material at $50 \%$ compressive strain may be caused by: i) fracture of the nanofibres and ii) separation of the nanofibres from PHEMA matrix. In order to validate this mechanism of failure, SEM examinations on the texture of BC-PHEMA hydrogel was conducted under $20 \%$ and $50 \%$ compressive strain and results are shown in Figure $7 \mathrm{a}$ and $7 \mathrm{~b}$. These specimens were prepared through freeze-drying method to remove the water content from the hydrogels, hence, the stressed status of specimens can maintained during the freeze-drying process. The crosssections of the specimens were made after the freeze-drying process followed by the observation using SEM. From Figure 7a, the original porous structure (Figure 2f) has been compressed and condensed due to the $20 \%$ compressive strain, but the nanofibres are still underneath the PHEMA matrix. Under $50 \%$ of compressive strain (Figure $7 \mathrm{~b}$ ), the microstructure of the BC-PHEMA hydrogel was crumbled and the nanofibres emerged from the underneath of PHEMA. Table 3 listed the comparison of simulation results and experimental results in this study. The results observed from Figure $7 \mathrm{~b}$ was supported by the simulation that the large stress difference existed in the material under large 50\% strain (Figure 6f), and such large stress difference has caused the separation of the nanofibres from PHEMA matrix. Therefore, the fracture of the reinforced hydrogel BC-PHEMA under compression is primarily due to the separation of nanofibres and PHEMA matrix.

Table 3. Summary of Simulation results Versus Experimental observation

\begin{tabular}{clll}
\hline Compressive strain & \multicolumn{1}{c}{ From 0 to ultimate } & \multicolumn{1}{c}{$20 \%$} & \multicolumn{1}{c}{$50 \%$} \\
\hline \multirow{2}{*}{ Simulation results } & Dashed lines in Figure 5 & $\begin{array}{l}\text { Figure 6c \& 6d. Internal } \\
\text { stress is evenly } \\
\text { distributed }\end{array}$ & $\begin{array}{l}\text { Figure 6e \& 6f. Interface } \\
\text { between nanofibres and } \\
\text { PHEMA is highly } \\
\text { stressed }\end{array}$ \\
\cline { 3 - 4 } Experimental results & Solid lines in Figure 5 & $\begin{array}{l}\text { Figure 7a. Microporous } \\
\text { structure is squeezed, } \\
\text { no fracture observed. }\end{array}$ & $\begin{array}{l}\text { Figure 7b. Fracture } \\
\text { observed: nanofibres } \\
\text { separated from PHEMA. }\end{array}$ \\
\cline { 2 - 4 } Conclusions & $\begin{array}{l}\text { Mooney-Rivlin theory } \\
\text { can describe the } \\
\text { mechanical behaviour of } \\
\text { PHEMA, BC, and BC- }\end{array}$ & $\begin{array}{l}\text { Microstructure inside } \\
\text { BC-PHEMA is } \\
\text { recoverable under small } \\
\text { deformation. }\end{array}$ & $\begin{array}{l}\text { Highly stressed interface } \\
\text { causes separation of } \\
\text { fibres and PHEMA, } \\
\text { thereby cause material } \\
\text { failure. }\end{array}$ \\
\hline
\end{tabular}




\section{Conclusions}

As a new class of nanofibre-reinforced hydrogels, a tough and strong PHEMA-based nanofibre reinforced hydrogel (BC-PHEMA) which is synthesised through a mixture of artificial hydrogel (PHEMA) and natural hydrogel (bacterial cellulose) has been intensively investigated in this paper. At microscale level, SEM observation has confirmed that the BC-PHEMA hydrogel exhibits porous structure with $0.57 \mu \mathrm{m}$ average diameter of pores. As the result of the microstructural change, the mechanical properties of such reinforced hydrogel have been significantly enhanced; for instance, the ultimate tangent modulus of the BC-PHEMA can three times and ten times higher than PHEMA and $\mathrm{BC}$, respectively. According to the inter-relationship between tangent modulus and the compressive strain, the deformation and fracture behaviour of the reinforced hydrogel BC-PHEMA have to be considered at microscale level, given the complex microstructure due to $\mathrm{BC}$ nanofibres present in the PHEMA matrix. To elaborate the fracture physics and fundamental mechanism of mechanical behaviour of the BC-PHEMA hydrogel under compression, numerical simulations demonstrated its usefulness in deriving various data to describe the deformation process based on Mooney-Rivlin hyperelastic theory. The curve fitting through simulation has been performed, and the results showed a strong agreement to the experimental data. The simulation which are strongly supported by experimental results indicates the potential deformation mechanism of BC-PHEMA under the compression, which are involved: i) within small deformation/strain (<20\%), the PHEMA matrix was primarily subject to the compressive stress against the compression loading; ii) When the deformation/strain is over $20 \%$, nanofibres were also under the compression being subject to certain compressive stress, in such way, the reinforcement of PHEMA matrix through BC nanofibres may be achieved; and iii) the fracture of the BC-PHEMA hydrogel as the results of high compression strain (strain $\geqq 50 \%$ ) is hardly recoverable which is primarily attributed to the high degree of separation of nanofibres from PHEMA matrix.

\section{Acknowledgement}

The authors would like to thank the 7th European Community Framework Program for financial support through a Marie Curie International Research Staff Exchange Scheme (IRSES) Project, entitled "Micro-Multi-Material Manufacture to Enable Multifunctional Miniaturized Devices (M6)," 
(Grant No. PIRSES-GA-2010-269113).

\section{References}

1 Gibas I, Janik H. Review: synthetic polymer hydrogels for biomedical applications. Chemistry and chemical technology. 2010, 4(4):297-304.

2 Butcher JT, Nerem RM. Porcine aortic valve interstitial cells in 3D culture: Comparison of phenotype with aortic smooth muscle cells. J Heart Valve Dis, 2004, 13:478-485

3 Jang J, Lee J, Seol YJ, Jeong YH, Cho DW. Improving mechanical properties of alginate hydrogel by reinforcement with ethanol treated polycaprolactone nanofibres. Compos Part B-Eng, 2013. 45(1), 1216-1221.

4 Kerin AJ, Wisnom MR, Adams MA, The compressive strength of articular cartilage. Proc Instn Mech Engrs Part H, 1998. 212, 273-280.

5 Hu J, Hiwatashi K, Kurokawa T, Liang SM, Wu ZL, Gong JP. Micro-reinforced hydrogel films with high mechanical strength and their visible mesoscale fracture structure. Macromolecules, 2011, 44(19), 7775-7781.

6 Cheung H, Ho M, Lau K, Cardona F, Hui D. Natural fibre-reinforced composites for bioengineering and environmental engineering applications. Compos Part B-Eng, 2009, 40(7), 655-663.

7 Roman J, Cabanas MV, Pena J, Vallet-regi M. Control of the pore architecture in three-dimensional hydroxyapatite-reinforced hydrogel scaffolds. Sci Technol Adv Mat, 2011, 12, 045003.

8 Zhou CC, Ye XJ, Fan YJ, Qing FZ, Chen HJ, Zhang XD. Synthesis and characterization of CaP/Col composite scaffolds for load-bearing bone tissue engineering. Compos Part B-Eng, 2014, 62, 242248.

9 Agrawal A, Rahbar N, Calvert PD. Strong fibre-reinforce hydrogel. Acta Biomaterialia, 2013, 9:53135318.

10 Qin Y, The preparation and characterization of fiber reinforced alginate hydrogel. J Appl Polym Sci. 2008, 108(5), 2756-2761.

11 Young CD, Wu JR, Tsou TL. High-strength, ultra-thin and fibre-reinforced PHEMA artificial skin. Biomaterials. 1998, 1745-1752.

12 Hassan CM, Peppas NA. Strucutre and applications of poly(vinyl alcohol) hydrogels produced by conventional crosslinking or by freezing/thawing methods. Adv. Polym. Sci. 2000, 37-65.

13 Hestrin S, Schramm M, Synthesis of cellulose by acetobacter xylinum. II. Preparation of freezedried cells capable of olymerizing glucose to cellulose. Biochem J. 1954, 58(2):345-352.

14 Shi Z, Phillips G O, Yang G. Nanocellulose electroconductive composites. Nanoscale. 2013, 5(8): 3194-3201.

15 Nakayama A, Kakugo A, Gong JP, Osada Y, Takai M, Erata T, Kawano S. High mechanical strength double-network hydrogel with bacterial cellulose. Adv. Funct. Mater. 2004, 14(11), 1124- 
1128.

16 Matinez RV, Fish CR, Chen Z, Whitesides GM, Elastomeric Origami: Programmable paperelastomer composites as pneumatic actuators. Adv. Funct. Mater. 2012, 22, 1376-1384.

17 Zhao W, Liu C, Lenardi C, Santaniello T, Wu F. Mechanical fastening to enable room temperature packaging for LOCs based on biocompatible hydrogel thin film. Proceeding of the 14th International Conference on Electronics Materials and Packaging. Hong Kong, China, 2012. pp. 316-320.

18 Zhao W, Liu C, Wu F, Lenardi C. An investigation on the mechanical behaviour of poly(2hydroxyethyl methacrylate) hydrogel membrane under compression in the assembly process of microfluidic system. J. Polym. Sci. B: Polym. Phys.. 2014, 52, 485-495. DOI: 10.1002/polb.23449

19 Clayton AB, Chirila TV, Dalton PD. Hydrophilic Sponges Based on 2-Hydroxyethyl Methacrylate. III. Effect of Incorporating a Hydrophilic Crosslinking Agent on the Equilibrium Water Content and Pore Structure. Polym. Int.. 1997, 42(1): 45-56.

20 Lou Z, Dalton PD, Chirila TV. Hydrophilic sponges based on 2-hydroxyethyl methacrylate part VII: Modulation of sponge characteristics by changes in reactivity and hydrophilicity of crosslinking agents. J. Mater Sci.: Mater Med. 2000, 11(5):319-325.

21 Martello F, Tocchio A, Tamplenizza M, Gerges I, Pistis V, Recenti R, Bortolin M, Fabbro MD, Argentiere S, Milani $P$, Lenardi C. Poly(amido-amine)-based hydrogels with tailored mechanical properties and degradation rates for tissue engineering. Acta Biomaterialia. 2014, 10(3), 12061215.

22 Calvert P. Hydrogels for soft machines. Adv. Mater. 2009, 743-756.

23 Peppas NA and Benner RE, Proposed method of intracordal injection and gelation of poly(vinyl alcohol) solution in vocal cords: polymer considerations. Biomaterials, 1980, 1(3), 158-162.

24 Kristi SA, Christopher NB, Lisa BP. Review: Mechanical properties of hydrogels and their experimental determination. Biomaterials, 1996, 17, 1647-1657.

25 Zhao W, Lenardi C, Webb P, Liu C, Santaniello T, Gassa F. A methodology to analyse and simulate mechanical characteristics of poly(2-hydroxyethyl methacrylate) hydrogel. Polym Int, 2013, 62, 1059-1067.

26 Rivlin RS. Large elastic deformations of isotropic materials. IV. Further developments of the general theory. Philosophical Transactions of the Royal Society, 1948, A 241(835), 379-397.

27 Klemm D, Schumann D, Udhardt U, Marsch S. Bacterial synthesized cellulose-artificial blood vessels for microsurgery. Prog. Polym. Sci.. 2001, 26:1561-1603.

28 Samir M, Alloin F, Dufresne A, Review of recent research into cellulosic whiskers, their properties and their application in nanocomposite field. Biomacromolecules, 2005. 6:612-626.

29 Iguchi M, Yamanaka S, Budhiono A. Bacterial cellulose- a masterpiece of nature's arts. J Mater Sci 2000; 35:261-270.

30 Verna C, Melsen B, Melsen F, Differences in static cortical bone remodeling parameters in human mandible and iliac crest. Bone, 1999. 25(5):577-83. 
31 Stein MS, Feik SA, Thomas CD, Clement JG, Wark JD. An automated analysis of intracortical porosity in human femoral bone across age. J Bone Miner Res. 1999. 14(4):624-32.

$32 \mathrm{Li}$ Y, Wang S, Huang R, et al. Evaluation of the effect of the structure of bacterial cellulose on full thickness skin wound repair on a microfluidic chip. Biomacromolecules, 2015, DOI: 10.1021/bm501680s

33 Anssari-Benam A, DL Bader, Screen HRC. A combined experimental and modelling approach to aortic valve viscoelasticity in tensile deformation. J Mater Sci: Mater Med. 2011, 22:253-262.

34 Screen HRC, Lee DA, Bader DL, Shelton JC. An investigation into the effects of the hierarchical structure of tendon fascicles on micromechanical properties. Proc Insts Mech Engrs Part H: J Eng Med. 2004, 218,:109-119.

35 McCutchen CW, Lubrication of Joints, in The Joints and Synovial Fluid (Ed: Sokoloff L), Academic, New York 1978, Chapter 10, 437-483.

36 Obeid EMH, Adams MA, Newman JH, Mechanical properties of articular cartilage in knees with unicompartmental osteoarthritis, J. Bone. Joint. Surg.[Br], 1994, 76-B(2):315-319. 